\title{
Katholische Presse - Brücke zur Solidarität*
}

\author{
von Karl R. Höller
}

Auf meinem Schreibtisch steht seit Wochen eine Postkarte mit einer Karikatur, die mir deshalb so großen Spaß macht, weil sie das überzogene Sendungsbewußtsein der Werbebranche auf die spitze Feder nimmt. Zu sehen ist da ein junger Mann - offenbar Werbetexter von Beruf -, der an einem Schreibtisch voller Gummibärchen über dem treffenden Slogan brütet. Vor ihm steht, mit erhobenem Zeigefinger, sein Chef und sagt per Sprechblase: „Merken Sie sich, Mann, wir verkaufen keine Gummibärchen, wir haben eine Message."

Die Geschichte geht noch weiter. Als ich mich auf dieses Referat vorbereitete, schaute mir meine Tochter über die Schulter, sah das Thema und meinte trocken: „Da steckst Du aber ganz schön in der Message drin!“ Übrigens eine schonende Ausdrucksweise; denn in ihrem 17jährigen Umgangsdeutsch hätte dies normalerweise gelautet: „Da haben sie Dir aber ein Laber-Thema angedreht."

Katholische Presse - Brücke zur Solidarität, das ist fast ein so schöner Pleonasmus wie die Kirchenzeitungs-Überschrift „Papst für den Frieden“. Allerdings enthält die von mir erwartete Eingrenzung des Themas auf Weltkirche, Dritte Welt und universelle Solidarität so viele sinn- und hoffnungsvolle Aspekte, daß ich mir erlaube, das Thema um ein kleines Wort zu ändern. Ich stelle die These auf, katholische Presse sei nicht nur eine Brücke zur, sondern eine Brücke der Solidarität. „Man kann sich nur schwer vorstellen", sagt die Pastoralinstruktion „Communio et progressio“", "wie jemand das Gebot Christi treu erfüllen will, wenn er die Vorteile und Chancen der Kommunikationsmittel, einer außerordentlich großen Zahl von Menschen die Lehren und Gebote des Evangeliums zu bringen, ungenutzt läßt.“

Die katholische Presse der Bundesrepublik hat diese Chance genutzt. Sie ist wesentlich daran beteiligt, daß sich der Gedanke der Verantwortung fürund der Brüderlichkeit mit den Menschen in den sogenannten Entwicklungsländern und den jungen Kirchen tief in unseren Gemeinden verankerte. Sie hat auch entscheidenden Anteil an den RekordKollekten der großen Werke Misereor, Adveniat und Missio. Wäre ich dogmatischer Theologe, würde ich diese Behauptung nicht nur zur „sententia pia et probabilis“, sondern „de fide“ erklären. Statistisch belegen läßt sie sich allerdings nicht. Dazu schweigt bisher die publizistikwissenschaftliche Literatur ebenso wie die sonst so gewissenhafte Buchführung der Hilfswerke.

Einem Bericht über die Pressearbeit des Werkes Missio ${ }^{2}$ entnehme ich, daß im Laufe des Jahres 1984 - das Jahr ist zufällig - annähernd 100 Nachrichten, Reportagen, Kommentare und Interviews von der Missio-Pressestelle über KNA verbreitet wurden. Etwa 30 Meldungen gingen zusätzlich über die Deutsche Presse-Agentur. 83 Reportagen liefen über die Foto-Agenturen KNA-Bild und foto-present. Man wagt es kaum, diese Daten auf alle Werke hochzurechnen und sie mit den Eigenbeiträgen der Kirchenpresse oder den Informationen aus den Orden zu addieren.

* Vortrag anläßlich der Jahrestagung der Arbeitsgemeinschaft Katholische Presse am 6.9.1989 in Essen, dokumentiert in der AKP-Dokumentation, Bonn, 1989/1 


\section{Brücke der Solidarität: Ergebnisse der Meinungsforschung}

Fragen wir einleitend verschiedene Meinungstests, ob sie verläßliche und realistische Auskunft geben können, wie es um die katholische Presse als „Brücke der Solidarität“ bestellt ist. Bei Befragungen kommen verständlicherweise nur die Rezipienten zu Wort, doch sie spielen, wie wir gelernt haben, im publizistischen Funktionsmodell keine geringere Rolle als die Kommunikatoren. ${ }^{3}$ Noch recht widersprüchlich antwortet die groBe Synodenumfrage aus dem Jahre 1971 . $^{4}$ Sie wollte von den deutschen Katholiken wissen, worüber auf der Synode unbedingt gesprochen werden müßte. Knapp ein Drittel (29,9\%) der Befragten war der Meinung, daß die „Verantwortung unserer Kirche für die Kirche in anderen Ländern und für die Weltmission “ unbedingt dazugehöre. Andererseits machten $80,3 \%$ ein dickes Kreuz hinter die ihnen vorformulierte Überzeugung, es sei besonders wichtig, "daß die Kirche die Botschaft Gottes in aller Welt verkündet“. - "Beide Antworten zusammengenommen waren ein Signal dafür, daß einerseits die Bereitschaft zu missionarischem Engagement vorhanden war, andererseits jedoch ein hoher Prozentsatz der Katholiken sich der veränderten Situation und Aufgabenstellung im Bereich der Weltmission, die dringend einer Aufarbeitung bedurften, offenbar nicht bewußt war. ${ }^{\text {s }}$

Mehr ins Detail geht die Feldbefragung der Kirchenpresse im Jahre $1975 .{ }^{\circ} 55 \%$ der regelmäßigen Teilnehmer am kirchlichen Leben halten die "Religiöse Entwicklung in der Welt“ für ein sehr wichtiges Thema und möchten zu 61 \% regelmäßig darüber informiert werden. $\mathrm{Zu} 66$ \% glaubt man die eigene Informationserwartung durch die Kirchenpresse abgedeckt, doch nur zu $33 \%$ durch die Tagespresse.

Ermutigend sind auch die Zahlen zur Information über die Entwicklungsprobleme in der Dritten Welt, wiederum bezogen auf die regelmäßigen Kirchgänger. Informationsbedarf sehr wichtig: $36 \%$; Informationserwartung regelmäßig: $37 \%$; effektive Informationsabdeckung durch die Kirchenpresse: $59 \%$.

Die höchsten Erwartungsdaten erhält die kirchliche Weltmissionsarbeit. Informationsbedarf sehr wichtig: $52 \%$; Informationserwartung regelmäßig: $58 \%$; effektive Informationsabdeckung durch die Kirchenpresse: $73 \%$. Schauen wir uns dagegen das Thema „Weltwirtschaftliche Entwicklung “ an, halten es $51 \%$ für sehr wichtig, doch nur $18 \%$ wollen regelmäßige Information, und nur $19 \%$ finden ihr Informationsbedürfnis durch die Kirchenpresse abgedeckt, aber 76 \% durch das Fernsehen.

Vergleicht man die letzteren Daten mit den wesentlich günstigeren zum Thema „Entwicklungsprobleme in der Dritten Welt“, verläßt einen der ohnehin schon schwache Glaube an die Götter der Demoskopie, die - wie im alten Griechenland - oft genug die Gestalt derer annehmen, die sie beschwören.

Dennoch müssen wir der Vollständigkeit halber die Funktionsanalyse der Bistumsblätter aus dem Jahr 1987 heranziehen? ${ }^{7}$. Da fragen die Demoskopen, warum die Kirchenzeitung hauptsächlich gelesen wird. $21 \%$ der Abonnenten und $9 \%$ der Nichtabonnenten lesen sie "wegen der Glaubenshilfen“, nur $5 \%$ der Abonnenten und $4 \%$ der Nichtabonnenten hingegen wegen der Berichte aus der Weltkirche. Blättern wir nur drei Seiten weiter, erkundigen sich die Meinungsforscher nach den Themen, zu denen die Kirchenzeitung Stellung nehmen sollte. Mit $71 \%$ bei den Abonnenten und $69 \%$ bei den Nichtabonnenten stehen „Ehe und Familie“ auf dem ersten Platz, diesmal dicht gefolgt vom Thema "Dritte Welt" ( $50 \% / 51 \%$ ). Meinungsforscher können sich auf die offensichtliche Diskrepanz einen wortreichen Reim machen, aber ist dieser wissenschaftliche Reim mehr als Dichtung? 
Sie bemerken meine Skepsis gegenüber Meinungsbefragungen aller Art, die eine grundlegende Kurskorrektur von Gesinnungs-Publizistik einleiten sollen. Das mag angehen beim Abtasten von Zeitgeist-Themen und bei der Erfüllung der angenehmsten publizistischen Bedürfnis-Befriedigung. Wo es jedoch um Überzeugung geht, ist allein Überzeugung in der Lage, Überzeugung zu vermitteln. Bevor ich mich daher auf eine endlos-kontroverse und wegen der fehlenden Belege letztlich fruchtlose Diskussion einlasse, flüchte ich mich in die Geschichte. Sie ist ein verläßlicherer Beleg.

\section{Brücke der Solidarität: Belege der Geschichte}

Folgen Sie mir zurück an die Wende des 18. zum 19. Jahrhundert. Bis zu diesem Zeitpunkt war die Versorgung der Missionsländer - „Entwicklungsländer“ kannte man noch nicht - ureigenste Angelegenheit der Kirche und ihrer Orden. Seit dem 15. Jahrhundert übernahmen auch die Kolonialmächte Spanien und Portugal diese Verpflichtung. Französische Revolution, Napoleons Säkularisation, die Aufhebung der Klöster, das Verbot des Jesuitenordens sowie der Niedergang der iberischen Mächte ließen das gesamte System kollapsieren. „Sowohl bezüglich des Missionspersonals als auch der Katholikenzahl war die katholische Mission zu Beginn des 19. Jahrhunderts auf dem absoluten Tiefpunkt angelangt. ${ }^{\text {" }}$ In allen damals bekannten Weltteilen scheint es kaum mehr als 300 Missionare gegeben zu haben. ${ }^{9}$

Auf diesen Tiefpunkt folgte unmittelbar, konsequent und kontinuierlich die wohl dynamischste Epoche der Kirchengeschichte. Innerhalb von 150 Jahren wurde aus der Heidenmission die Weltmission, und aus der Weltmission erwuchs die Weltkirche. Zwei Faktoren, von der Geschichtsschreibung bislang kaum beachtet, haben entscheidend zu dieser Entwicklung beigetragen: die missionarische Laienbewegung des 19. Jahrhunderts und deren integrativ-solidarisches Informationsverhalten.

Als die königlichen und fürstlichen Spenden versiegten, wurde auf geradezu providentielle Weise die Missionssache zur Volkssache. Diese historische Wende steckt voller hochinteressanter Details, die ich mir versagen muß, um beim Thema zu bleiben. Nur soviel: Am 3. Mai 1822 gründete die junge Französin Pauline Jaricot mit Freunden in Lyon den „Verein der Glaubensverbreitung “. Ihre Devise: „Kleine Beiträge, aber von vielen; ein tägliches kleines Missionsgebet, aber von Millionen. "Sie erfand ein Organisationsprinzip, das durch Einfachheit und Genialität bestach: Die Mitglieder wurden in Zehner-, Hunderter- und Tausendergruppen organisiert. Einen wesentlichen Anreiz zum Beitritt - und damit sind wir wieder mitten in unserem Thema - bildeten die „Annalen“, eine periodische Publikation mit Berichten aus und über die Missionsländer. Sie zirkulierten in den Zehnergruppen und wurden von deren Leitern aufbewahrt.

Wie ein Flächenbrand - das abgenutzte Bild ist hier angebracht - breitete sich die Idee dieses Vereins über Frankreich und die Nachbarländer aus, erreichte um 1840 schon Amerika, 1848 Asien und 1857 Afrika. Die Annalen erschienen bald in vielen Sprachen.

Mit dem Ereignis von Lyon fällt 1821 der Beginn der deutschsprachigen katholischen Presse als einer bewußt und bekennend als katholisch auftretenden Publizistik zusammen, und zwar durch die Gründung der Zeitschrift „Der Katholik" in Mainz ${ }^{10}$. Dieser „Katholik“ erwies sich bereits 1829 als Brücke der Solidarität über die Grenzen hinaus, indem er in einem längeren Beitrag für die Teilnahme an der Vereinigung für die Ausbreitung des Glaubens zu Lyon warb. 
Damals gab-es noch keinen deutschen $Z$ weig. Doch bei dessen Gründung griff wenige Jahre später die Pressepublizistik ebenfalls entscheidend ein. Anläßlich eines Krankenbesuchs im belgischen Montzen entdeckte der Aachener Arzt Dr. Heinrich Hahn auf dem Nachttisch seines Patienten ein Heft der französischen „Annalen“. Er las und wurde spontan Mitglied. Bereits 1832 gelang es ihm, in Aachen einen Freundeskreis um sich zu sammeln, der die über ganz Deutschland verstreuten Missionsvereine nach dem Muster von Lyon und in Beziehung zu Lyon zusammenfassen und dem Werk eine gesetzliche Form geben wollte."

Das wiederum ist die Geburtsstunde unserer MISSIO für den Bereich der Fuldaer Bischofskonferenz. Die Bayern gingen - wie bekannt - einen eigenen Weg. Deutschsprachige Übersetzungen der "Annalen“ gab es nach 1832 schon in Einsiedeln, Straßburg, Köln und München.

Schauen Sie kurz mit mir hinein in die Geschichtsschreibung des 19. Jahrhunderts. Sie finden ganze Bibliotheken über den politischen und sozialen Katholizismus. Das weltkirchlich-missionarische Engagement hat nur wenige Beobachter. Es vollzieht sich gewissermaßen subkutan - unter der Haut des offiziellen Katholizismus - und wird doch letztlich unabsehbare weltkirchliche Konsequenzen haben.

Die Entwicklung geht weiter. Man darf davon ausgehen, daß die Zugehörigkeit zum Glaubensverein und die regelmäßige Lektüre der „Annalen“ in den Familien ein Klima schufen, in dem auch Missionsberufe reifen konnten. So bereitete die Laienbewegung mit ihrer Motivationskraft an der Basis die Wiederbelebung des aktiven missionarischen Dienstes in Deutschland vor. Was wir heute mit Stolz „Weltkirche" nennen, ist durch eine Vielzahl missionarischer Gemeinschaften im aktiven Dienst grundgelegt worden: 1875 wurde die Steyler Missionsgesellschaft vom Göttlichen Wort gegründet; 1881 entstand die Gesellschaft der Salvatorianer; 1884 die Missionsabtei der Benediktiner von St. Ottilien; 1892 eröffneten die Pallottiner ein Haus in Limburg, 1895 die Oblaten in Hünfeld. Die Liste der Gründungen ist keineswegs vollständig und läßt sich bis zum Ausbruch des ersten Weltkriegs weiterführen.

Doch noch einmal zurück zum publizistischen Engagement des deutschen Glaubensvereins. Er löste 1917 die alten „Annalen“ durch die Zeitschrift „Weltmission“ ab, wurde 1922 vom Franziskus-Xaverius-Missionsverein zum Päpstlichen Werk der Glaubensverbreitung, taufte sich 1972 in Missio um und führt die Zeitschrift „Weltmission“ seit 1969 als die Farbillustrierte „Mission aktuell“" mit einer der höchsten Auflagen innerhalb des katholischen Pressewesens weiter. Die alte „Weltmission“ wurde am 27. August 1937 durch die Geheime Staatspolizei verboten. Es gibt keinen besseren Beweis ihres solidarischen Charakters als die Begründung dieses Verbots: „Infolge der kitschigen und unwahrhaften Art der Darstellung der Verhältnisse bei fremden Völkern und durch die Verherrlichung fremder Rassen gegenüber den Europäern ist das Verbot der genannten Zeitschrift notwendig geworden."

Bei Nennung der missionarischen Gemeinschaften, die in der zweiten Hälfte des 19. Jahrhunderts entstanden, muß bei vielen von Ihnen lebendige Pressegeschichte angeklungen sein. Die meisten dieser Gründungen sind ohne publizistische Begleitaktivitäten nicht zu denken. Davon zeugen heute noch Titel, die in der Arbeitsgemeinschaft Katholische Presse Klang und Namen haben. Stellvertretend für viele soll hier das Steyler Pressewesen stehen, das älter ist als das Steyler Missionswerk. Bevor er einen Orden gründete, hatte Arnold Janssen 1874 mit dem „Kleinen Herz-Jesu-Boten“ bereits eine Zeitschrift $^{12}$, mit der er sowohl die Anliegen der ,inneren“ wie der „äußeren“ Mission vertrat. „In unseren Tagen“, schreibt er, „ist die Presse eine Großmacht ... Sie muß mit einem Schwert verglichen werden, das man im Kampf der Geister führt. " ${ }^{13}$ Kaum je- 
mand hat die Presse so konsequent für den solidarischen Dienst an den Menschen benutzt wie dieser Ordensgründer und nachfolgend seine „Gesellschaft des Göttlichen Wortes“. Ich erinnere nur an das Phänomen der "Stadt Gottes“, das längst eine intensive wissenschaftliche Behandlung verdient hätte. Rund um den Erdball entstanden Steyler Druckereien. Insgesamt werden heute von den Steyler Missionaren etwa 70 Zeitungen und Zeitschriften herausgegeben, redigiert oder gedruckt. ${ }^{14}$

Bevor wir das 19. Jahrhundert und damit die Frühgeschichte der katholischen Presse als einer solidarischen Mittlerin zur weltweiten Mission verlassen, sei daran erinnert, daß die Zahl der Titel dieses spezifischen Genres 1910 weit größer war als heute. 29 Zeitschriften der verschiedensten Gemeinschaften sind für dieses Jahr 1910 verbürgt. ${ }^{15}$

\section{Brücke der Solidarität: Weltoffenheit der katholischen Kirchenpresse nach 1945}

Gerne entschuldige ich mich bei denen, die sich mit historischen Darstellungen langweilen. Doch manchmal sind sie unverzichtbar. Die heutige Weltkirche mit der Mehrheit der Katholiken auf der südlichen Erdhalbkugel ist wirklich nicht zu verstehen ohne den missionarischen Aufbruch der Laien im 19. Jahrhundert, und dieser hätte nicht die dynamische Wirkung gehabt ohne die integrative Kraft ihrer Publizistik. Von Mission ist die Rede, nicht von Entwicklungsarbeit. Diesen Begriff gab es noch nicht, aber vieles, was wir heute darunter verstehen, war im missionarischen Dienst integriert. Auch die Presse, von der wir sprechen, ist nur ein Segment der Kirchenpresse, nämlich die heimische Missionspresse. Es gibt kaum Belege dafür, daß sich auch andere kirchliche Zeitungen und Zeitschriften programmatisch des Themas annahmen, von gelegentlichen Ausnahmen abgesehen. Möglicherweise ist das der Grund, weshalb die Geschichtsschreibung die missionarische Laienbewegung nie in den Blick bekam.

Selbst in den Jahren zwischen den Weltkriegen taucht „Mission“ in kirchlichen Sonntagsblättern, die eher Erbauliches bringen, nur unter erbaulichen Aspekten auf: in Geschichten über die Bekehrung des Kannibalen oder die Abkehr des Polygamisten von seiner frevlerischen Lebensweise.

Erst nach 1945 sind die Voraussetzungen dafür geschaffen, daß sich die kirchliche Presse zur Dritten Welt hin öffnet. Katholische Tagezeitungen gibt es - bis auf wenige Ausnahmen - nicht mehr. Die diözesanen Sonntagsblätter sind bereits zur Nazizeit in den Überlebensschutz des einen Bistumsblattes geflohen, das nun von den Sonntagsblättern das Erbauliche, von der früheren Zentrumspresse aber auch die Verpflichtung zu einer gewissen aktuellen Berichterstattung übernimmt.

Dennoch dauert es bis zum Ende der fünfziger Jahre, daß die Missionsländer als Entwicklungsländer mit ihren ungeheuren Problemen an Krankheit, Hunger und Unterernährung in den Blick kommen, - und damit auch in die Spalten der Kirchenpresse. Die Kolonialzeit ist vorbei. Ein Volk nach dem anderen - vor allem in Afrika - kämpft um die Unabhängigkeit. Das macht aufmerksam und wird auch über die säkularen Medien vermittelt. In seiner berühmten Rede vor der Vollversammlung der deutschen Bischöfe vom 15. bis 21. August 1958 in Fulda, die zur Gründung des Werkes Misereor führte, hat Kardinal Frings die Situation treffend beschrieben ${ }^{16}:{ }_{n}$ Erst seitdem uns ... die fernen Länder nahe gekommen sind, tritt uns ihre Not 'vor Augen'. Was wir bisher gewußt haben, 'sehen' wir jetzt. Was wir bisher über unserer eigenen Not vergessen ha- 
ben, tritt jetzt in die Mitte unseres Bewußtseins: in den meisten Ländern dieser Erde herrscht Hunger.“ Der Kardinal nennt in seiner Rede auch die Quellen dieser bedrükkenden Information: „Die kirchliche und katholische Presse beginnt eindrucksvolle Einzelheiten zu bringen ..." ${ }^{17}$

Von nun an ist das Thema im kirchlichen Pressewesen fest verankert. Seit der Vorbereitung der ersten Misereor-Fastenkollekte 1959 bleibt es - mit wechselnden Betonungen auf Barmherzigkeit, Entwicklungshilfe, Entwicklungsverantwortung oder Entwicklungszusammenarbeit - wie kaum ein anderes auf der Tagesordnung der katholischen Presse, sogar so nachdrücklich, daß der missionarische Dienst im Bewußtsein der Katholiken zurückgedrängt wird. Erst in den siebziger Jahren pendelt sich das Verhältnis von Leib- und Heilssorge wieder ein auf die biblisch und theologisch gesunde Relation, - bis hin zum Theorem ihrer Identität.

Fast jedes kirchliche Wochenblatt und jede Kirchenzeitung hat fortan eine eigene Dritte-Welt-Geschichte. Keine kirchliche Publikation verschloß sich grundsätzlich der publizistischen Vorbereitung und Begleitung der Kampagnen der großen Werke. Viele taten wesentlich mehr: Der Solidaritätsbogen reicht von gezielten Spendenaufrufen über den Brückenschlag zu den Missionaren aus dem eigenen Bistum (hier denke ich an Münster) bis zur Gründung kleiner, nicht ganz uneigennützig auch der Leserbindung dienender Hilfswerke.

Statt vieler Einzelbelege lassen Sie mich den evangelischen Pfarrer und Publizisten K. Rüdiger Durth mit einem Zeitschriftenbeitrag aus dem Jahre 1980 zitieren $^{18}$ : „Daß die Leser von Bistumsblättern und anderen katholischen Zeitschriften bereit sind, tief in die Tasche zu greifen, um mit 'ihrer' Zeitung Not zu lindern, zeigen einige Zahlen aus dem Jahr 1978: Die 'Neue Bildpost', die über ein eigenes Hilfswerk verfügt, konnte so zwei Millionen DM einsetzen. Vom Urwaldhospital in Kenia angefangen bis hin zur Flüchtlingshilfe in Thailand. Die Leser der „Münchener Katholischen Kirchenzeitung “ brachten 256.000,-- DM für Missionare, über 100.000,-- DM für Behinderte, 100.000,-- DM für Einsame und Arme sowie 60.000,-- DM für die Erhaltung einer südtiroler Kirche auf. Der 'Pilger' aus Speyer konnte 360.000,-- DM seiner Leser für die Entwicklungshilfe der katholischen Kirche zur Verfügung stellen, und die Leser des Katholischen Sonntagsblattes Rottenburg haben bis 1978 insgesamt 900.000,-- DM zur Errichtung einer Heimstatt geistig behinderter Kinder bereitgestellt."

Mitglieder der Arbeitsgemeinschaft Katholische Presse und der Gesellschaft katholischer Publizisten gründen am 24. Januar 1983 in Aachen das Heinrich-Jansen-CronWerk. Zweck des Vereins ist die Förderung und Ausbildung von Publizisten in Lateinamerika, Asien und Afrika, z. B. durch Stipendien; die Förderung von Kommunikation untereinander sowie die Unterstützung von in Not geratenen Publizisten und deren Familien in der Dritten Welt.

Katholische Presse - Brücke zur/der Solidarität, - ich glaube, das sind Belege genug. Dennoch möchte ich eine Initiative besonders hervorheben, weil sie sich von anderen abhebt und Auswirkungen hatte, von denen der Initiator selbst nichts ahnte. Viele werden sich an die "Ruhrwort"-Aktion „Der Groschen macht's" in den sechziger Jahren erinnern. „Ruhrwort“-Chefredakteur Dr. Otto Kaspar verfolgte mit seiner Aktion, die im dritten Jahr 470.000,-- DM aufbrachte, nicht Unterstützung allgemeiner Entwicklungsaufgaben. Er sah die Priorität seines Engagements im Aufbau der katholischen Presse - Solidarität zur Vermittlung von Solidarität - in der Dritten Welt. Dafür bat er seine Leser zur Kasse, die bereitwillig spendeten. Lieblingsidee von Otto Kaspar war eine „Medien-Feuerwehr“, zusammengestellt aus journalistischen Fachleuten der ver- 
schiedensten Ordensgemeinschaften, die überall dort eingesetzt werden sollten, wo es um die sinnvolle Neugründung einer Zeitung, einer Druckerei oder einer Hörfunkstation ging. Auf Kaspars Initiative hin gründete die UCIP, die Internationale Union der katholischen Presse, eine eigene Entwicklungskommission. Statt der „Medienfeuerwehr“ entsprang Kaspars unablässigem Drängen 1969 die Gründung des „Catholic Media Council“ in Aachen. Unter dem gemeinsamen Dach von internationalen katholischen Medienorganisationen und Hilfswerken steht Cameco heute mitten in einem weltweiten Erfahrungsaustausch, plant, koordiniert, prüft, dokumentiert und berät. Um diese Fachstelle wird die katholische Kirche von vielen Organisationen beneidet. Es dürfte keine zweite Einrichtung geben, die so detailliert über publizistische Vorgänge in der Dritten Welt unterrichtet ist. ${ }^{19}$

Hier wäre der Ort, etwas über die Bedeutung der Pressepublizistik und ihre Solidaritätsfunktion in den Ländern Afrikas, Asiens und Lateinamerikas selbst zu sagen. Sie werden es mir erlassen; denn das Thema ist nun doch zu weit. Es reicht von der Errichtung der ersten Druckerei in Mexiko 1539 bis zur Rolle der katholischen Presse bei der sogenannten „Rosenkranz-Revolution“ aufden Philippinen 1987. Die entsprechenden Unterlagen füllen beim Catholic Media Council ganze Aktenschränke. Was im Grunde zu sagen wäre, faßt das berühmte Dokument der lateinamerikanischen Bischofsversammlung von Medellin aus dem Jahr 1968 für diesen Kontinent wie folgt zusammen: „Ohne Massenmedien kann man weder den Lateinamerikaner in seiner Menschenwürde heben noch die notwendigen Reformen des Kontinents in Gang bringen. Das heißt aber, daß es nicht nur nützlich und schicklich ist, sondern unbedingt notwendig, die Massenmedien auf allen Ebenen und in allen Bereichen seelsorglicher Tätigkeit einzusetzen, will man die von dieser unserer Versammlung gesteckten Ziele erreichen. ${ }^{20}$

\section{Brücke der Solidarität: Das geistige Konzept}

In seiner bereits zitierten Rede zur Gründung von Misereor skizziert Kardinal Frings auf bewundernswert weitsichtige Weise auch das geistige Konzept des neuen Werkes. Es soll nicht nur darum gehen, Barmherzigkeit zu üben, Hilfe zu geben und Unterentwickelte zu entwickeln. Vielmehr ,tut sich hier die Möglichkeit auf, dem praktischen Materialismus praktisch, d.h. durch ein religiöses Tun, zu begegnen, nämlich in einem freiwilligen Verzicht auf Güter dieser Welt um der Not Christi willen “. ${ }^{21}$ Dem Fasten einen neuen Sinn geben und darüber hinaus der gesamten Öffentlichkeit das objektive Unrecht der ungleichmäßigen Verteilung der Güter dieser Welt vor Augen stellen - das ist angesagt. Die Brücke der Solidarität soll von Beginn an auf zwei tragenden Pfeilern hüben und drüben - errichtet werden.

Dafür braucht es die Unterstützung der katholischen Presse, die vorbehaltlos gegeben wird. Allerdings taten sich alle Beteiligten noch recht schwer mit dem Brückenbau. Man dachte intentional, war fixiert auf Armut und Not in der Dritten Welt, wollte die Gewissen der Reichen wachrütteln und die Geldbeutel öffnen. Dabei wurden alle Register der Mitleidsorgel gezogen. Kein Elendsbild konnte hart genug, keine Situationsschilderung drastisch genug sein. Zu dieser Zeit - es war zu Beginn der 60er Jahre - arbeitete ich als junger Redakteur bei einer Kirchenzeitung. Das Thema der Entwicklungshilfe finanzierte uns. Die Anliegen des Pastoralwerks Adveniat für Lateinamerika wurden stillschweigend darunter subsumiert. Wir gaben dem massiven Kampf gegen Hunger, Krankheit und Unterentwicklung unser eigenes strategisches Konzept. In un- 
serem Übereifer merkten wir nicht, daß wir dabei waren, die Mitmenschen aus Afrika, Asien oder Lateinamerika am unteren Ende eines drastisch dramatisierten Miserabilitäts-Gefälles als ewige Hilfsempfänger festzuschreiben.

So gesehen haben wir in den letzten 30 Jahren einen gewaltigen Lernprozeß durchgemacht: von der Hilfe zur Selbsthilfe, vom Slogan „Millionen heißen Lazarus“ zum Leitwort „Anders leben“, von der Darstellung des unterentwickelten zum geistig und kulturell gleichwertigen oder sogar überlegenen Partner, vom Lehren zum Lernen, vom Schenken zum Beschenktwerden. Am Ende steht die Einsicht: Es gibt keine unterentwickelten Menschen.

In den siebziger Jahren hat sich für diesen Lernprozeß, dem wir Journalisten ebenso unterzogen waren wie unsere Rezipienten, der Begriff der „Bewußtseinsbildung “ eingebürgert. Er ist nicht besonders schön und wurde vielfach abgelehnt, weil man ihn mit dem Konzept der „conscientizacao“ des Lateinamerikaners Paulo Freire verwechselte. ${ }^{22}$ Zentraler methodischer Ansatz Freires ist die Hinführung unterprivilegierter Gruppen und Personen zur Erfahrung ihrer eigenen, meist gesellschaftlich bedingten, vielfältigen Frustrationen. Da „Bewußtsein und Welt simultan“ sind, soll aus der Selbsterfahrung, aus Frustration und sogar Aggression die Veränderung der Gesellschaft erwachsen.

Ganz anders verstehen die beiden Synodendokumente zu Mission und Entwicklung die Bedeutung der Bewußtseinsbildung für die Dritte Welt. Zwar sollen auch hier die Zusammenhänge der Unterentwicklung einsichtig gemacht und Informationen vermittelt werden, doch wird die eigentliche Veränderung durch Glaubensvertiefung, Gewissensbildung und persönliche Kontakte erreicht. ${ }^{23}$

Die Terrorismusforschung der 70er Jahre hat gezeigt, wie gefährlich es sein kann, Frustrationen bewußt zu machen und dann in Initiativen gesellschaftlicher Veränderung umzusetzen. Sehr viel guter Wille, dem fernen Nächsten solidarisch beizustehen, schlug um in resignativ zerstörerische Wut, weil sich offensichtlich in Chile oder Südafrika trotz allen Engagements doch nichts ändern ließ.

Die katholische Presse hat mitgeholfen, ein völlig anderes Modell kirchlicher Bildungsarbeit für die Dritte Welt zu propagieren. Es geht aus von der ärgerlichen Tatsache, daß wesentliche Aspekte des Status und des Prestiges einer Konsumgesellschaft wie in der Bundesrepublik die wirtschaftliche Eigenständigkeit und Sicherheit sind. Die Menschen der Dritten Welt erscheinen „der Hilfe bedürftig“; weil sie diesen Status noch nicht erreicht haben. Ein solches Denken endet in der Sackgasse. Deshalb dreht kirchliche Bildungsarbeit die Wertehierarchie um. Wirtschaftliche Fragen haben hinter geistigen und kulturellen Werten zurückzustehen. Mag der Partner noch so arm sein, mag seine Hütte auf Pfählen stehen, - diese Pfähle wurzeln im Boden uralter Kulturen und religiöser Überlieferungen. Es wächst eine neue Spendenmotivation aus dem Wunsch nach Rückkoppelung vitaler Lebens- und Glaubensvollzüge der Menschen in der Dritten Welt, von denen wir lernen können. Das Stichwort dieser Spendenmotivation heißt „positive Partnerschaft“. Erlauben Sie mir, in diesem Zusammenhang Emil Dovifat, den Nestor der deutschen Publizistikwissenschaft, zu zitieren, der kurz vor seinem Tode schrieb: „Nichts lähmt mehr als das eisige Grau ewigen Nörgelns ... Der größte Publizist des hohen Mittelalters, Bernhard von Clairvaux, hat dies gemeint, wenn er in eine sich verzehrende Zeit hineinrief: Mögen andere schelten, ich will bewundern! “ ${ }^{44}$

Wir erleben, wie sich unter solchen Vorzeichen der vorübergehend weggetauchte missionarische Auftrag der Kirche zurückmeldet. In den Prozeß des geistigen Austauschs 
hat er mehr einzubringen als der Entwicklungsdienst. Aus früheren Missionskirchen sind inzwischen eigenständige Ortskirchen geworden mit einer überraschenden Vielfalt an religiös-kulturellen Ausdrucksweisen.

\section{Brücke der Solidarität: Blick in die Zukunft}

Damit richten wir den Blick in die Zukunft. Brücke zur Solidarität - was heißt das für die kirchliche Presse heute und morgen? Nach wie vor meint Solidarität die Bereitschaft zur materiellen Hilfe. Dafür muß die kirchliche Presse antreten wie am ersten Tag ihres jetzt schon mehr als 30jährigen Engagements. Im Bereich der Entwicklungsarbeit und im Einsatz für die Menschenrechte sind die Vorzeichen eher düsterer geworden. In seiner Sozialenzyklika „Sollicitudo rei socialis“ sagt Papst Johannes Paul II. sehr offen, daß die anfangs so lebhaften Hoffnungen auf Entwicklung heute weit entfernt von ihrer Verwirklichung erscheinen. Das objektive Unrecht, von dem Kardinal Frings 1958 sprach, besteht unzweifelhaft fort. Kirchliche Entwicklungshilfe wird nie ausreichen, um die großen Ungleichgewichte zwischen Nord und Süd, die schreienden Ungerechtigkeiten im Weltwirtschaftssystem, in den Finanzströmen und in den Handelsstrukturen zu beseitigen. Aber sie kann politischen Entscheidungen, wann immer sie fallen werden, die richtigen Wege vorzeichnen.

Solches zu verdeutlichen, ist Auftrag der kirchlichen Presse. Nach offensichtlicher Verschwendung von Ressourcen, nach unheilvoller Verquickung von Außen- und Entwicklungspolitik, geht es darum, der prioritär armutsorientierten Entwicklungshilfe das Wort zu reden. Geld muß wieder dort ankommen, wo es notwendig ist, wo es Not wendet. Die Forderung der frühen Entwicklungsarbeit nach Hilfe zur Selbsthilfe bekommt eine neue Umschreibung:

- Maßnahmen der integrierten ländlichen Entwicklung haben Vorrang vor Großprojekten;

- in städtischen Elendsvierteln ist das Gemeinwesen auszubauen;

- das Gesundheitswesen muß dezentralisiert werden, damit eine medizinische Grundversorgung der Armen gewährleistet ist;

- bei Bildungsmaßnahmen stehen praxisorientierte berufliche Aus- und Fortbildung vor formellen Ausbildungsprogrammen. ${ }^{25}$

Wer solche Programme gegen den anhaltenden Trend von Entwicklungsprojekten der Großmannssucht durchsetzen will, braucht breite Rückendeckung durch Sympathisanten und Spender sowie ständigen Flankenschutz durch die katholische Presse.

Das alles gilt erst recht für die Akzeptanz der Kriterien, die unsere kirchliche Entwicklungsförderung heute auf ihre Projekte anwendet. Diese Kriterien zeigen, daß man hinter den materiellen Bedürfnissen der Menschen in der Dritten Welt die Notwendigkeit zur ganzheitlichen Entwicklung sehen muß:

- Entwicklungsprojekte des neuen Typs müssen sozialverträglich sein. Schädliche soziale Nebenwirkungen für verarmte geselischaftliche Gruppen sind im vorhinein zu vermeiden.

- Entwicklungsprojekte müssen umweltverträglich sein. Sie dürfen das ökologische Gleichgewicht nicht zusätzlich stören. Dahinter steht die Forderung nach einer neuen Ethik im Umgang mit natürlichen Ressourcen. 
- Entwicklungsprojekte müssen kulturverträglich sein. Sie sind keine für Christen vertretbare Hilfe, wenn sie zum Bruch mit den Traditionen und Kulturen einer Gesellschaft führen oder verführen. ${ }^{26}$

Aus all dem ersehen Sie, daß die katholische Presse als Brücke der Solidarität in Zukunft mehr gefordert sein wird als in den Jahren, die hinter uns liegen, - und was wir von der Solidarität im Entwicklungsdienst gesagt haben, ist noch eindringlicher gefordert für die innerkirchliche Kommunikation einer sich täglich verändernden Weltkirche.

Die Fakten sind bekannt. Schon rein quantitativ hat sich das Schwergewicht der katholischen Kirche und des gesamten Christentums zunehmend von Europa und Amerika zu den Ländern der Südhalbkugel verlagert. Um die Jahrtausendwende werden zwei Drittel aller Katholiken in den früheren Missionsländern leben. „Die europäischabendländische Kirche muß heute feststellen, - und diese Umorientierung ist noch keineswegs abgeschlossen -, daß gewisse, noch nicht ganz geschwundene Dominanzansprüche über die Ortskirchen des Südens allenfalls historisch zu erklären, aber durch

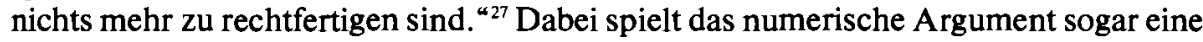
untergeordnete Rolle. Als eigenständige Ortskirchen durch das Zweite Vatikanische Konzil dazu ermutigt, sind die Mitchristen der Dritten Welt das Wagnis der Inkulturation eingegangen. Sie befragen ihre eigene geistig-religiöse Tradition nach Hilfen zum Ausloten des offenbarten Gotteswortes. Neue Theologien entstehen. Schauen Sie einmal hinein in ein Heft der „Theologie im Kontext “ aus dem Missionswissenschaftlichen Institut Missio, und Ihnen wird schwindlig, was sich weltweit tut. Theologen warnen schon jetzt, daß aus Asien eine Christologie auf uns zukomme, der gegenüber die Auseinandersetzung mit der lateinamerikanischen Befreiungstheologie ein Kinderspiel gewesen sei. ${ }^{28}$

Hans Meier glaubte auf einer der letzten Vollversammlungen des Zentralkomitees der deutschen Katholiken feststellen zu müssen, Rom halte Europa nur noch für eine sekundäre Größe im Weltkatholizismus und sei inzwischen ganz auf die sogenannten jungen Kirchen fixiert. Er fand Widerspruch. Auch ich halte wenig davon, das Christentum in der Dritten Welt zu idealisieren. Auch dort gibt es Fehler und Versagen, und es sind Zweifel erlaubt, ob uns in gleichem Maße gut bekommt, was in Afrika oder Asien den Glauben belebt. Wir werden nie rhythmisch um den Altar tanzen oder in der Essener Münsterkirche mit Andacht die Baumtrommel schlagen.

Etwas anderes aber ist lebenswichtig. Die Entwicklung geht so schnell, daß wir in Gefahr sind, den Anschluß zu verlieren. Wir müssen voneinander wissen. Wir müssen verstehen können, warum Afrikaner oder Inder so und nicht anders denken. Sonst zersplittert die weltkirchliche Gemeinschaft, auf die wir so stolz sind, bevor sie sich festigen konnte. Die Koinonia, die Communio der Kirchen, verwirklicht sich nicht bloß in der vertikalen Dimension, in der Gemeinschaft mit Rom, sondern auch in der horizontalen Dimension, im Austausch der Kirchen untereinander.

Wer soll diesen Austausch leisten, wenn nicht die Kirchenpresse? Sagen Sie nicht, solche Themen seien zu theoretisch und wenig pressegerecht. Es zeichnet doch gerade die Dritte-Welt-Theologie aus, daß sie narrativ ist. In mancher Geschichte und in manchem Gebet steckt tieferer theologischer Gehalt als in geschwätzigen Büchern, die wir unseren Lesern anpreisen! 
Der Missionstheologe Walbert Bühlmann ${ }^{29}$ hat die Impulse, auf die wir zu achten haben, in griffige Formeln gefaßt:

- für Lateinamerika: Den Glauben tun!Verkopfte Theologie ändert wenig am Leben. In kleinen christlichen Gemeinschaften wird der Glaube getan, und erst später reflektieren Theologen darüber.

- für Afrika: Den Glauben feiern! „Glaube ist ja nicht ein Müssen, sondern ein Dürfen, nicht eine langweilige Pflichtübung, sondern ein froh stimmendes Angebot." Wer feiern kann, sagt Ja zum Leben und kehrt als ein anderer vom Fest zurück.

- für Asien: Vom Glauben her die Welt deuten! „Glauben heißt ja nicht, abstrakte Worte und Formeln anzunehmen, sondern den letzten Sinn des ganzen Weltgeschehens deuten, die ganze Welt gehalten wissen in der Hand des geheimnisvollen Gottes."

Sagen Sie nicht: Wo bekommen wir die Informationen her? Die katholische Kirche ver-

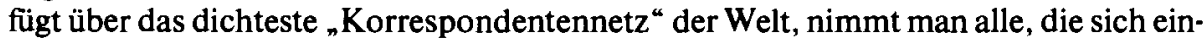
ander mitteilen können. Außerdem ist der Kirchenpresse zu empfehlen, in diesen Fragen die Werke etwas mehr in die Pflicht zu nehmen.

Noch gar nicht gesprochen haben wir vom Aufbruch und der Renaissance der großen Weltreligionen, die sich vor unserer eigenen Haustüre ereignet. Nicht gesprochen haben wir davon, daß sich Kirchenpresse in den Dialog mit diesen Weltreligionen einschalten muß; denn das Fremde und die anderen sind so lange eine Gefahr, als man sich ihnen entzieht und sich nicht auf sie einläßt.

Wir leben zweifellos in einer der interessantesten Epochen der Kirchengeschichte. Was sich in der Weltkirche tut, ,was unter der Bezeichnung 'Inkulturation' theologisch und praktisch verhandelt wird, gehört zu den erregendsten Erscheinungen unserer Zeit“", sagt Hans Waldenfels. ${ }^{30}$

Vor einem solchen Horizont müßte so manches von dem zusammenschrumpfen, was wir heute für publizistisch wichtig halten. Theologengezänk oder nörgelnde innerkirchliche Kritik, denen wir die Schlagzeilen geben, werden später einmal - wenn überhaupt - das Kleingedruckte in den Büchern der Kirchengeschichte ausmachen. Guter erlauben Sie mir das Wort: prophetischer - Journalismus muß das später Großgedruckte vorausahnen, und dazu zählt zweifellos der gegenwärtige weltkirchliche Umbruch.

Lassen Sie mich enden mit einem Gedicht, das unser Thema noch einmal von ganz anderer Seite beleuchtet. Auf der Generalversammlung des Weltrates der Kirchen 1983 im kanadischen Vancouver legte auch die Arbeitsgruppe Kommunikation ihr Ergebnis vor. Sie hatte - wie selbstverständlich - die Verse eines indonesischen Delegierten aufgenommen, die er als seinen Diskussionsbeitrag ablieferte:

"In der Tiefe des Schweigens

sind Worte nicht nötig,

muß Sprache nicht sein.

In der Tiefe des Schweigens

bin ich zu hören gerufen. ${ }^{\star 31}$

„Christliche Kommunikation muß ankündigen,

Nein, christliche Kommunikation muß anzeigen,

Nein, christliche Kommunikation muß Teilen fördern,

Nein, christliche Kommunikation muß Gemeinschaft schaffen,

$\mathrm{Ja}$, christliche Kommunikation muß voll der Hoffnung sein..." 


\section{Anmerkungen}

1 Pastoralinstruktion „Communio et progressio", Nr. 126

2 Aktennotiz P $10 \mathrm{Ba} / \mathrm{Qu} 229$ vom 15.5.1985

3 vgl. Michael Schmolke, Fragen zum Ort von Gesinnungskräften im publizistischen Funktionieren, in: „Communicatio Socialis “, Emsdetten, 1. Jg. 1968, S. 109-115

4 Ludwig Wiedenmann, Einleitung: Missionarischer Dienst an der Welt, in: Gemeinsame Synode, Freiburg 1976, Seite 807

5 Ludwig Wiedenmann, a.a.O., S. 807

6 Institut für Kommunikationsforschung e.V., Wuppertal: Kommunikations- und Informationserwartungen der katholischen Bevölkerung gegenüber kirchlichen Massenmedien, Abschlußberichte 3 - 1975

7 Media Markt Analysen, Funktionsanalyse Bistumsblätter, Frankfurt 1987

8 Alphons Mulders, Missionsgeschichte, Regensburg 1960, S. 361

9 Mulders, a.a.O., S. 361

10 Michael Schmolke, Katholisches Pressewesen, in: Staatslexikon, Freiburg ${ }^{7} 1987$, Band 3, S. 365

11 Franz Baeumker, Dr. med. Heinrich Hahn - ein Apostel im Laienkleide, Aachen 1930, S. 327

12 Franz Josef Eilers, Arnold Janssen als Publizist: Ein Beitrag zur Geschichte der deutschsprachigen Missionspublizistik, in: „Communicatio Socialis“, Paderborn, 8. Jahrgang 1975, S. 301-323

13 Edmund Plazinski, Presse im Dienste des Wortes, in: Steyler Missions-Chronik 1975, Steyl, S. 129-131, hier: S. 129

14 Plazinski, a.a.O., S. 131

15 Robert Streit, Führer durch die deutsche katholische Missionsliteratur, Freiburg 1911

16 Leo Schwarz, Misereor, Zeichen der Hoffnung, München 1976, S. 13-34, hier S. 14

17 Leo Schwarz, a.a.O., S. 16

18 K. Rüdiger Durth, Die Anstrengungen lohnen sich: Die Katholische Presse in der Gegenwart (III), in: „Communicatio Socialis“, Paderborn, 13. Jahrgang, Nr. 2/1980, S. 105-118, hier S. 115

19 Karl R. Höller, Publizistische Medienplanung für Entwicklungsländer "Catholic Media Council“, in: „Communicatio Socialis“, Paderborn, 5. Jahrgang 1972, S. 57-63

20 Erklärung von Medellin 1968, 3.15

21 Dams u.a., Misereor a.a.O., S. 21

22 Paulo Freire, Pädagogik der Unterdrückten, Stuttgart-Berlin 1972

23 Hans Czarkowski, Psychologische A spekte der kirchlichen Bewußtseinsbildung für die Dritte Welt, in: „Communicatio Socialis", Paderborn, 10. Jg. 1977, S. 1-18, hier S. 4

24 Emil Dovifat, Der katholische Publizist, in: „Communicatio Socialis“, Emsdetten, 1. Jg. 1968 , S. 26-28, hier S. 27

25 siehe Norbert Herkenrath, Mehr als ein Tropfen auf den heißen Stein - Rechenschaft über 30 Jahre kirchliche Entwicklungshilfe, Manuskript eines Vortrags in Bonn vom 11.1.1989

26 Norbert Herkenrath, Mehr als ..., a.a.O., Manuskript S. 4

27 Klaus Nientiedt, Zuflucht Dritte Welt, in: Herder Korrespondenz, Freiburg, 40. Jg. 1986, Heft 8, S. 350

28 Walbert Bühlmann, Impulse aus den Jungen Kirchen, in: „Katechetische Blätter“, München, 9:1987, S. 706-715, hier S. 712

29 Walbert Bühlmann, Impulse ..., a.a.O., S. 709-715

30 Hans Waldenfels, Im Wettstreit um die Wahrheit, in: „Rheinischer Merkur“, Bonn, 44. Jahrgang 1989, Nr. 31, S. 22

31 Larry Jorgensen, Vancouver WCC General Assembly: Work group Communication, in: "Communicatio Socialis“, Paderborn, 16. Jg. 1983, S. 347-362, hier S. 359 